Division of General Internal Medicine and Clinical Innovation, New York University Langone Medical Center

Katherine.otto@nyulangone.org Twitter @kateotto

Cite this as: BMJ 2022;376:020 http://dx.doi.org/10.1136/bmi.o20

Published: 6 January 2022

\section{Centring equity over exceptionalism in the global covid-19 response}

An American primary care physician living in Venezuela grapples with the health inequities in covid-19 responses within and between her two countries

Katherine Otto Chebly adjunct faculty

The covid-19 pandemic has transformed arguments for global health equity from an altruistic rallying cry into a pragmatic imperative. As long as large clusters of people go without access to vaccines, variants like Omicron are more likely to develop and spread, continually disrupting the stability of public life. But for citizens of the global south, there is little to suggest that disparities in resource allocation will change anytime soon. To date, covid vaccines have been accessed by nearly $76 \%$ of people in high income countries and only $8 \%$ in low income countries. ${ }^{1}$

\section{Two countries, two sets of problems}

As an American primary care physician who is living and working in Venezuela, I am struck by the contrasting conversations happening about covid between my old and new homes. Healthcare worker colleagues in the US describe overwhelmed emergency departments and ICUs, their frustration with inadequate vaccination uptake, and the unrelenting moral injury they face from ceaseless dilemmas over how to care for people with covid-19. Alongside relief from new booster doses and paediatric vaccines comes simultaneous worry about eroding faith in healthcare institutions and deepening mistrust in medical leadership. Even within a well resourced nation, considerable threats to effective pandemic management remain.

Here in Caracas, our conversations are different. Colleagues work in an ecosystem void of data-rich Pfizer and Moderna vaccines, and instead manage with doses from Russia (Sputnik), China (Sinopharm, Sinovac), and Cuba (Abdala), not all of which are approved by the World Health Organisation, nor considered valid for international travel. While the ethics of vaccine mandates for healthcare staff are debated across the US, here healthcare workers in the public sector are eager for any available protection, as some contend with power outages, fuel shortages, and basic supply scarcities. Masks are donned, uncontested, in public spaces, as a cohesive act of community protection.

\section{Charity isn't the answer}

Despite the stark discrepancies in covid discourse, I see a shared struggle for health equity that connects my two worlds and, with it, an urgent need to prioritise pragmatism over even the most well intentioned acts of altruism. Consider that while over one billion donations to the Covid-19 Vaccines Global Access initiative (known as Covax) were announced in $2021,{ }^{2}$ less than 300 million vaccine doses have been delivered. ${ }^{3}$ These doses represent just a fraction of global need, and donations do not correct the fundamentally profit-driven motives of how covid If anything, the global north's adoption of a "saviour" role has only perpetuated dependent relationships, rather than nudging those who currently hold power towards true solutions-for example, pressuring pharmaceutical companies to share intellectual property with and produce vaccines in low income regions.

Meanwhile, nations left waiting for "gifts" lack clarity on if or when they will arrive, leaving them unable to reliably plan the logistics of vaccination campaigns, including adequate cold chain storage, sufficient equipment and staff, and the ability to time subsequent doses. Long delays also fuel public mistrust in and politicisation of vaccination campaigns, contributing to understandable waves of vaccine hesitancy.

\section{An ongoing struggle for equity}

Charity, in isolation, doesn't solve inequity; it solidifies systems of injustice. This lesson also applies to America's notoriously segregated healthcare system, which is rooted in socioeconomic and racial biases. When the covid-19 pandemic arrived in the US, lower income and more racially diverse communities experienced an excess burden of infections and deaths. ${ }^{4}$ Many of these patients, who were more likely to be uninsured or under-insured, lacked access to healthcare yet had to face the persistent risk of viral exposure in essential work roles.

Safety net hospitals, like the one in which I completed medical training, commonly work in close partnership with the vulnerable communities they serve, providing accessible, informed care so that unjust socioeconomic circumstances do not have to dictate health outcomes. During the first covid surge, my colleagues expertly tended to a tragic volume of covid admissions, ${ }^{5}$ while simultaneously providing care to prevent additional infections, including a robust telemedicine service that counselled and cared for thousands of ambulatory patients in their homes. 6 But community based health centres across the US remain underfunded, despite their long term commitment to otherwise marginalised patients and their strategic, sensible efforts to create healthier environments that benefit all citizens.

Managing the covid-19 pandemic in any part of the world requires an understanding that essential healthcare, including covid vaccines, should never be framed as gifts from the rich to the poor, but as entitlements in a world that values human life equally. We are all witnesses to this lesson: the whole vaccines are produced and where they are distributed. 
world suffers when scientific breakthroughs leave most of the world behind, or when high quality healthcare remains systemically inaccessible to some patients. The covid pandemic calls us to centre equity over exceptionalism, as a path that is not only morally robust, but also one on which we are all better off.

Competing interests: none declared.

Provenance and peer review: not commissioned, not peer reviewed.

1 Share of people who received at least one dose of COVID-19 vaccine. Our World in Data. 2021. https://ourworldindata.org/grapher/share-people-vaccinated-covid?country=High+income Upper+middle+income $\sim$ Lower+middle+income $\sim$ Low+income

2 COVID-19 vaccine doses donated to COVAX. Our World in Data. 2021. https://ourworldindata.org/grapher/covax-donations?coun-

try=FRA $\sim$ ESP $\sim$ SWE $\sim$ USA CAN NOR NZL GBR DNK CHE ITA DEU PRT $\sim$ ARE $\sim B E L \sim$ European+Union JPN NLD FIN HKG IRL

3 COVAX Vaccine Roll-out. Gavi: The Vaccine Alliance. 2021. https://www.gavi.org/covax-vaccineroll-out

4 Adhikari S, Pantaleo NP, Feldman JM, Ogedegbe O, Thorpe L, Troxel AB. Assessment of Community-Level Disparities in Coronavirus Disease 2019 (COVID-19) Infections and Deaths in Large US Metropolitan Areas. JAMA Netw Open 2020;3:e2016938.

doi: 10.1001/jamanetworkopen.2020.16938. pmid: 32721027

5 Uppal A, Silvestri DM, Siegler M, etal. Critical Care And Emergency Department Response At The Epicenter Of The COVID-19 Pandemic. Health Aff (Millwood) 2020;39:1443-9. doi: 10.1377/hlthaff.2020.00901. pmid: 32525713

6 Lau J, Knudsen J, Jackson H, etal. Staying Connected In The COVID-19 Pandemic: Telehealth At The Largest Safety-Net System In The United States. Health Aff (Millwood) 2020;39:1437-42. doi: 10.1377/hlthaff.2020.00903. pmid: 32525705 